\title{
Hohes Potenzial
}

\section{Neuer Therapieansatz in der Hepatologie}

— „Wir glauben, dass dies ein Durchbruch in der Behandlung der PSC sein könnte“, so die Einschätzung von Prof. Dr. Michael Trauner, Wien, mit Blick auf eine Studie zum Einsatz von norUrsodesoxycholsäure (norUDCA) bei primär sklerosierender Cholangitis (PSC). Die spannenden Ergebnisse wurden auf dem Internationalen Leberkongress der European Association for the Study of the Liver (EASL) präsentiert. Doch die Substanz hat noch mehr Potenzial.

\section{UDCA bei PBC etabliert}

UDCA (Ursofalk ${ }^{\circledast}$ ) ist als Standardtherapie bei primär biliärer Cholangitis (PBC) längst etabliert. Etwa zwei Drittel der Patienten zeigen ein biochemisches Ansprechen und können von einer an nähernd normalen Lebenserwartung ausgehen. Bei PSC zeigt UDCA dagegen keine überzeugende Wirksamkeit.

\section{Kleine Modifikation - große Wirkung}

Das scheint sich durch eine kleine chemische Modifikation, die Verkürzung einer Seitenkette, zu ändern. NorUDCA wird in der Leber gehalten und kann direkt auf die entzündeten Gallengänge wirken. Tierexperimentell zeigten sich ausgeprägte anticholestatische, antifibrotische und antiinflammatorische Effekte.

„NorUDCA stellt als Modifikation der UDCA einen vielversprechenden neuen Therapieansatz dar und unterscheidet sich von UDCA durch ihre besonderen physikochemischen Eigenschaften. Sie führen zu einer bikarbonatreichen Choverantwortlich ist.“ lerese, die für die protektiven Effekte

\section{Auch gegen die Fettleber?}

Neben der PSC sieht Trauner weitere Indikationen für norUDCA. Präklinische Daten, die eine Verbesserung der Fettleber zeigen, die von deutlichen antiatherosklerotischen Effekten begleitet werden, prädestinieren nach Trauner die modifizierte UDCA auch für die Therapie der nicht-alkoholischen Fettleber (non alcoholic fatty liver disease, NAFLD). Eine multizentrische Studie ist bereits auf den Weg gebracht. Zudem sieht Trauner norUDCA auch als mögliche Option bei Patienten mit PBC, die auf die etablierte Standardtherapie nicht ansprechen.

\section{Dr. Beate Fessler}

- Pressegespräch "Leber-und Darmerkrankungen - besserverstehen, gezielter behandeln"; Innsbruck, März 2016 (Veranstalter: Falk)

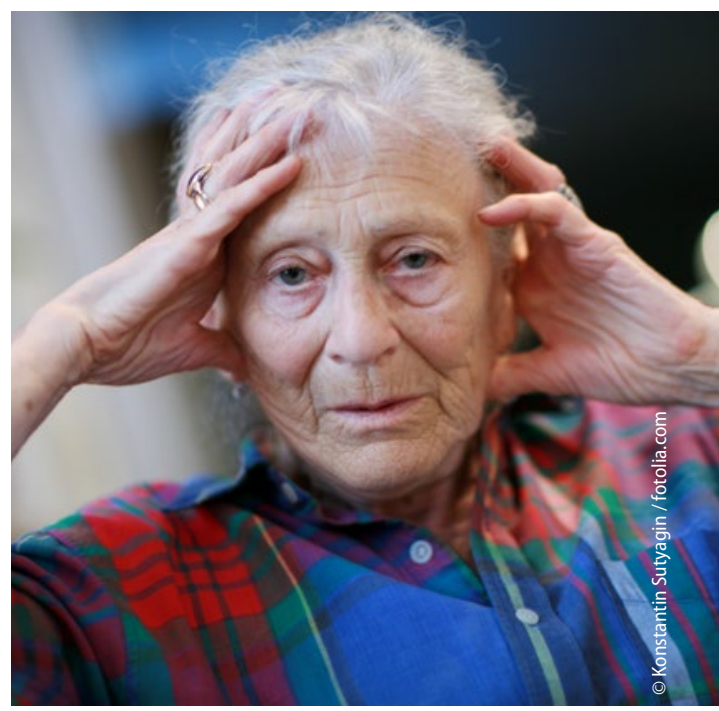

Gerade ältere Patienten können Hypoglykämien oft nicht richtig einordnen.

\section{Herzrhythmusstörung bis Demenz}

Schwere Hypoglykämien erhöhen das Risiko für Herzrhythmusstörungen, fördern arteriosklerotische Prozesse und steigern das Risiko für kardiovaskuläre Ereignisse, erklärte Prof. Werner Kern, Ulm. Zudem beeinträchtigten sie langfristig die kognitive Leistungsfähigkeit und erhöhten das Risiko für eine späte- re Demenz. Leichte und nächtliche Unterzuckerungen bemerke der Patient oft nicht - diese minderten aber Wohlbefinden und Arbeitsfähigkeit.

\section{Susanne Pick}

- Fachpressekonferenz „Herzlichen Glückwunsch-1 Jahr Insulin glargin U300", 51. Diabetes Kongress der Deutschen Diabetes Gesellschaft (DDG); Berlin, Mai 2016 (Veranstalter: Sanofi-Aventis) bessere Senkung des $\mathrm{HbA}_{1 \mathrm{c}}$-Wertes als nächtlicher schwerer oder bestätigter UnHypoglykämien zu jeder Tageszeit um Obes Metab. 2015]. Darüber hinaus biete das hoch dosierte Insulin den Patienten Flexibilität, denn es könne innerhalb eines Zeitfensters von sechs Stunden inauch für Diabetiker mit Nephropathie oder ältere Patienten, die besonders auf 